\title{
One neural networks model for Lyapunov matrix inequalities
}

\author{
Shiheng Wang ${ }^{1}$, Jia Sun ${ }^{2}$ and Ke Wang ${ }^{2, ~ *}$ \\ ${ }^{1}$ Nanyang Vocational College of Agriculture, Nanyang 473000, P.R. China \\ ${ }^{2}$ Department of Mathematics, Shanghai University, Shanghai 200444, P.R. China \\ the corresponding author, email: kwang@shu.edu.cn
}

Keywords: Zhang neural networks (ZNN), linear matrix inequality (LMI), Lyapunov matrix inequality.

Abstract. One kind of recurrent neural networks - Zhang neural networks (ZNN) is studied for solving a set of linear matrix inequalities - Lyapunov matrix inequalities.

\section{Introduction}

Linear matrix inequality (LMI) problems are widely encountered in numerous science and engineering applications [1]. For example, in multi-agent team cooperation in game theory, to avoid a lower cost for each agent at the expense of requiring full information set, additional constraints are added to the structure of the controller by using the linear matrix inequality (LMI) formulation of the minimization problem [2], where the following Lyapunov matrix inequality is involved,

$$
A^{\mathrm{T}} X+X A \leq B
$$

mostly in the time-varying form,

$$
A^{\mathrm{T}}(t) X(t)+X(t) A(t) \leq B(t)
$$

where $A(t) \in R^{n \times n}$ and $B(t) \in R^{n \times n}$ are smoothly time-varying coefficient matrices, assumed to be known numerically or could be estimated accurately, and $X(t) \in R^{n \times n}$ is the time-varying unknown matrix to be solved.

In this paper, a recurrent neural network - Zhang neural networks (ZNN) model is studied for solving time-varying Lyapunov matrix inequality (1.2). And numerical simulations are presented to show the excellent performance of the ZNN approach for Lyapunov matrix inequality (1.1).

\section{Lyapunov matrix inequality}

In this section, solving Lyapunov matrix inequality is converted to solving a Lyapunov matrix equation by introducing a time-varying matrix whose elements are non-negative.

The time-varying LMI (1.2) can be reformulated as

$$
f(X(t), t)=A^{\mathrm{T}}(t) X(t)+X(t) A(t)-B(t) \leq 0 \in R^{n \times n} .
$$

By introducing a time-varying matrix $\Lambda^{2}(t) \in R^{n \times n}$ whose elements are non-negative, the following time-varying matrix equation can be obtained,

$$
A^{\mathrm{T}}(t) X(t)+X(t) A(t)-B(t)+\Lambda^{2}(t)=0,
$$

where superscript ${ }^{2}$ denotes the square of the each element of a matrix. To solve (2.2), the following related definitions and lemmas are required.

DEFINITION 2.1 [3]. Given matrices $F=\left(f_{i j}\right) \in R^{m \times n}$ and $G=\left(g_{i j}\right) \in R^{p \times q}$, their Kronecker product (also termed, direct or tensor product) is denoted by $F \otimes G$ and is defined as the following block matrix,

$$
F \otimes G=\left(\begin{array}{ccc}
f_{11} G & \mathrm{~L} & f_{1 n} G \\
\mathrm{M} & \mathrm{O} & \mathrm{M} \\
f_{m 1} G & \mathrm{~L} & f_{m n} D
\end{array}\right) G \in R^{m p \times n q} .
$$


In general, $F \otimes G \neq G \otimes F$, and $F \otimes F^{\mathrm{T}} \neq F^{\mathrm{T}} \otimes F$ except $F=F^{\mathrm{T}}$.

DEFINITION 2.2 [3]. A matrix, $C=\left(c_{i j}\right) \in R^{p \times q}$, can be vectorized as the following by stacking all its column vectors,

$$
\operatorname{vec}(C)=\left(c_{11}, c_{21}, \mathrm{~L}, c_{p 1}, c_{12}, c_{22}, \mathrm{~L}, c_{p 2}, \mathrm{~L}, c_{1 q}, c_{2 q}, \mathrm{~L}, c_{p q}\right)^{\mathrm{T}} \in R^{p q \times 1} .
$$

LEMMA 2.1 [3]. If for any $t \geq 0$ there exists $\alpha>0$ such that

$$
\min \sigma\left(I \otimes\left(A^{\mathrm{T}}(t) A(t)\right)+A(t) \otimes A^{\mathrm{T}}(t)+A^{\mathrm{T}}(t) \otimes A(t)+\left(A^{\mathrm{T}}(t) A(t)\right) \otimes I\right) \geq \alpha,
$$

where $I$ is appropriate identity matrices and $\sigma(\cdot)$ denotes the eigenvalues set of a matrix, then the time-varying Lyapunov equation (2.2) has a unique solution.

A time-varying solution $X(t)$ and a time-varying $\Lambda(t)$ can be obtained by solving the time-varying Lyapunov matrix equation (2.2), then

$$
A^{\mathrm{T}}(t) X(t)+X(t) A(t)-B(t)=-\Lambda^{2}(t) \leq 0,
$$

which indicates that the LMI (1.2) can be solved via online solution of (2.2).

\section{Neural networks model}

In this section, the ZNN model is presented, developed and investigated for solving the time-varying Lyapunov matrix inequality (1.2).

The error function can be set as

$$
E(t)=A^{\mathrm{T}}(t) X(t)+X(t) A(t)-B(t)+\Lambda^{2}(t) .
$$

Therefore, the ZNN model can be established as follows [1],

$$
E(t)=\frac{\mathrm{d} E(t)}{\mathrm{d} t}=-\Gamma \Phi(E(t))
$$

where $\Gamma$ could be simply $\gamma I$ with constant scalar $\gamma>0$, and $\Phi(\cdot): R^{m \times n} \rightarrow R^{m \times n}$ denotes an activation function array of neural networks. In this paper, the function $\phi(\cdot)$, element of $\Phi(\cdot)$, is chosen as the power-sigmoid (p-s) activation function (with $p=3$ and $\xi=4$ ),

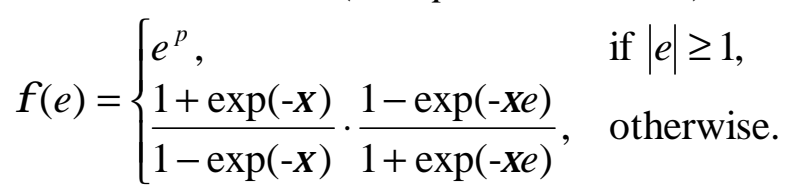

From (3.1) and (3.2), with the time-derivative of $E(t), \operatorname{vec}(\Lambda(t))=\left(\lambda_{1}(t), \lambda_{2}(t), \mathrm{L}, \lambda_{n^{2}}(t)\right)^{\mathrm{T}} \in R^{n^{2} \times 1}$, the Kronecker product and vectorization techniques, the following dynamic equation can be obtained,

$$
f(t)=M^{+}(t) P(t) y(t)+M^{+}(t) \operatorname{vec}(B(t))-M^{+}(t) \gamma \Phi(Q(t) y(t)-\operatorname{vec}(B(t))),
$$

where $y(t) \in R^{2 n^{2}}$ denotes the state of the neural network, and $M^{+}(t)$ denotes the pseudoinverse of $M(t)$, and the augmented matrices are denoted as follows,

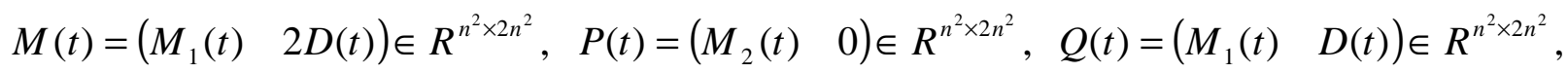

$$
\begin{aligned}
& M_{1}(t)=I \otimes A^{\mathrm{T}}(t)+A^{\mathrm{T}}(t) \otimes I \in R^{n^{2} \times n^{2}}, \quad M_{2}(t)=-\left(I \otimes \circledast \mathrm{T}(t)+\AA^{\mathrm{T}}(t) \otimes I\right) \in R^{n^{2} \times n^{2}}, \\
& D(t)=\operatorname{diag}\left(\lambda_{1}(t), \lambda_{2}(t), \mathrm{L}, \lambda_{n^{2}}(t)\right) .
\end{aligned}
$$

Starting from a random initial state $y(0) \in R^{2 n^{2}}$ and given an appropriate design parameter $\gamma>0$ as large as the hardware allows, the ZNN model (3.3) converges to a solution of time-varying Lyapunov LMI (1.2). The theoretical result is presented as follows.

THEOREM 3.1. Given smoothly $A(t) \in R^{n \times n}$ and $B(t) \in R^{n \times n}$ of time-varying Lyapunov matrix inequality (1.2), if a monotonically-increasing odd function array $\Phi(\cdot)$ is used, then the neural state $y(t)$ of ZNN model (3.3), starting from any initial state $y(0) \in R^{2 n^{2}}$, converges to a theoretical time-varying solution of (1.2). 


\section{Numerical example}

In this section, simulations are provided to illustrate the $\mathrm{ZNN}$ model by using different values of for solving time-varying Lyapunov matrix inequality (1.2).

EXAMPLE. Consider the problem of the time-varying Lyapunov matrix inequality (1.2) with constant coefficient matrices $A(t), B(t)$ as follows,

$$
A(t)=\left(\begin{array}{ccc}
0 & 1 & 0 \\
0 & 0 & 1 \\
-1 & -2 & -3
\end{array}\right), B(t)=\left(\begin{array}{lll}
1 & 0 & 0 \\
0 & 1 & 0 \\
0 & 0 & 1
\end{array}\right) .
$$

With $\operatorname{vec}\left(\Lambda^{2}(t)\right)=\left(\lambda_{11}{ }^{2}(t), \lambda_{21}{ }^{2}(t), \lambda_{31}{ }^{2}(t), \lambda_{12}{ }^{2}(t), \lambda_{22}{ }^{2}(t), \lambda_{32}{ }^{2}(t), \lambda_{13}{ }^{2}(t), \lambda_{23}{ }^{2}(t), \lambda_{33}{ }^{2}(t)\right)^{\mathrm{T}} \in R^{9}$, such a Lyapunov matrix inequality can be converted to a matrix equation (2.2). The proposed ZNN model (3.3), with the state vector $y(t)=\left(\operatorname{vec}(X(t))^{\mathrm{T}}, \operatorname{vec}(\Lambda(t))^{\mathrm{T}}\right)^{\mathrm{T}} \in R^{18}$, is used to solve the above Lyapunov matrix inequality as well as its converted matrix equation. The corresponding simulation results are shown in Figs. 1 and 2.
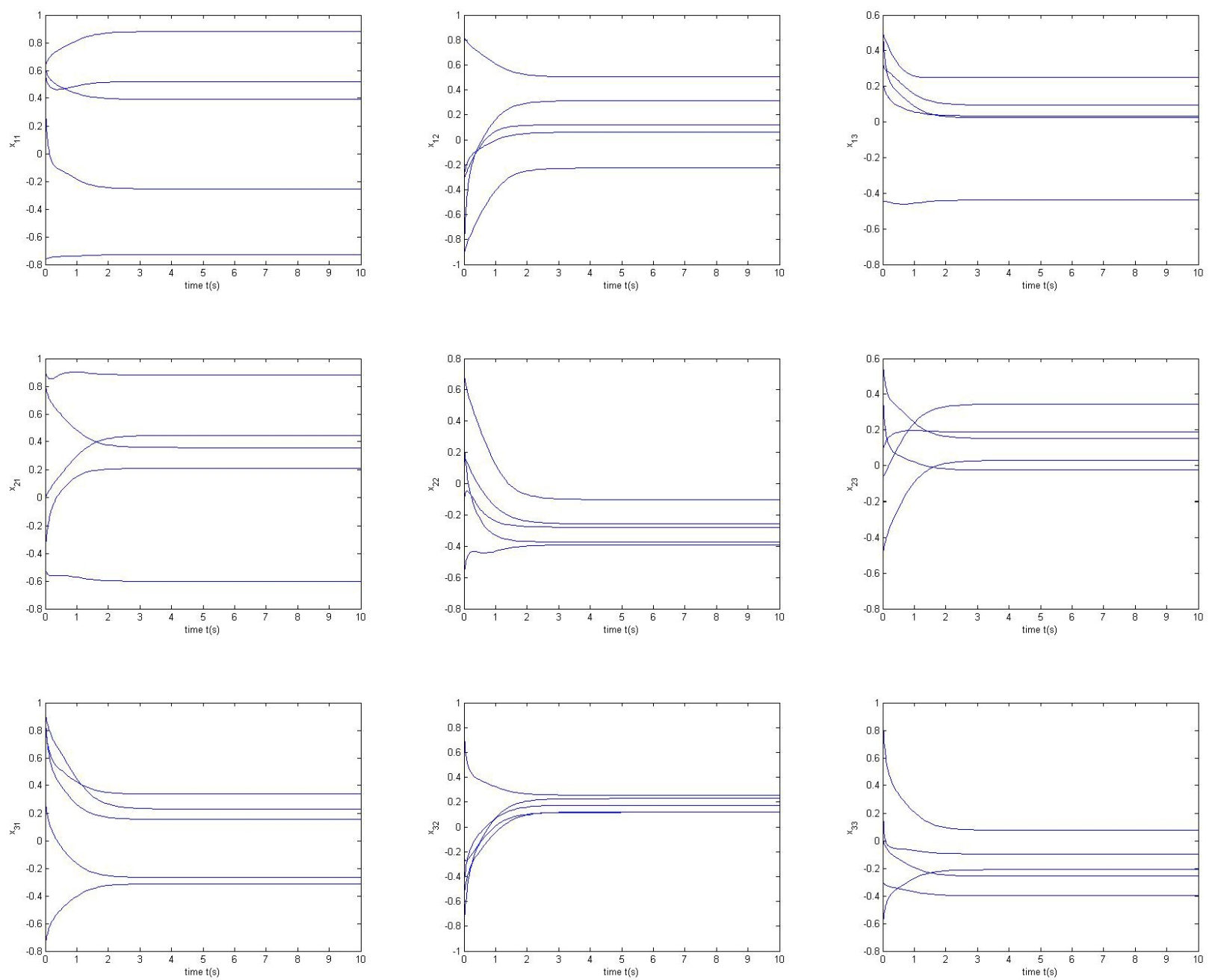

Fig. 1. State trajectories of $X(t) \in R^{2 \times 3}$ computed by the $\mathrm{ZNN}$ model (3.3) with $\gamma=1$ and using the power-sigmoid activation function array.

Fig. 1 illustrates the state trajectories synthesized by the proposed ZNN model (3.3) with $\gamma=1$ and using the power-sigmoid activation function array.

Fig. 2 shows that the residual errors of (3.3) (corresponding to Fig. 1) converge to zero within around $4 s$. 


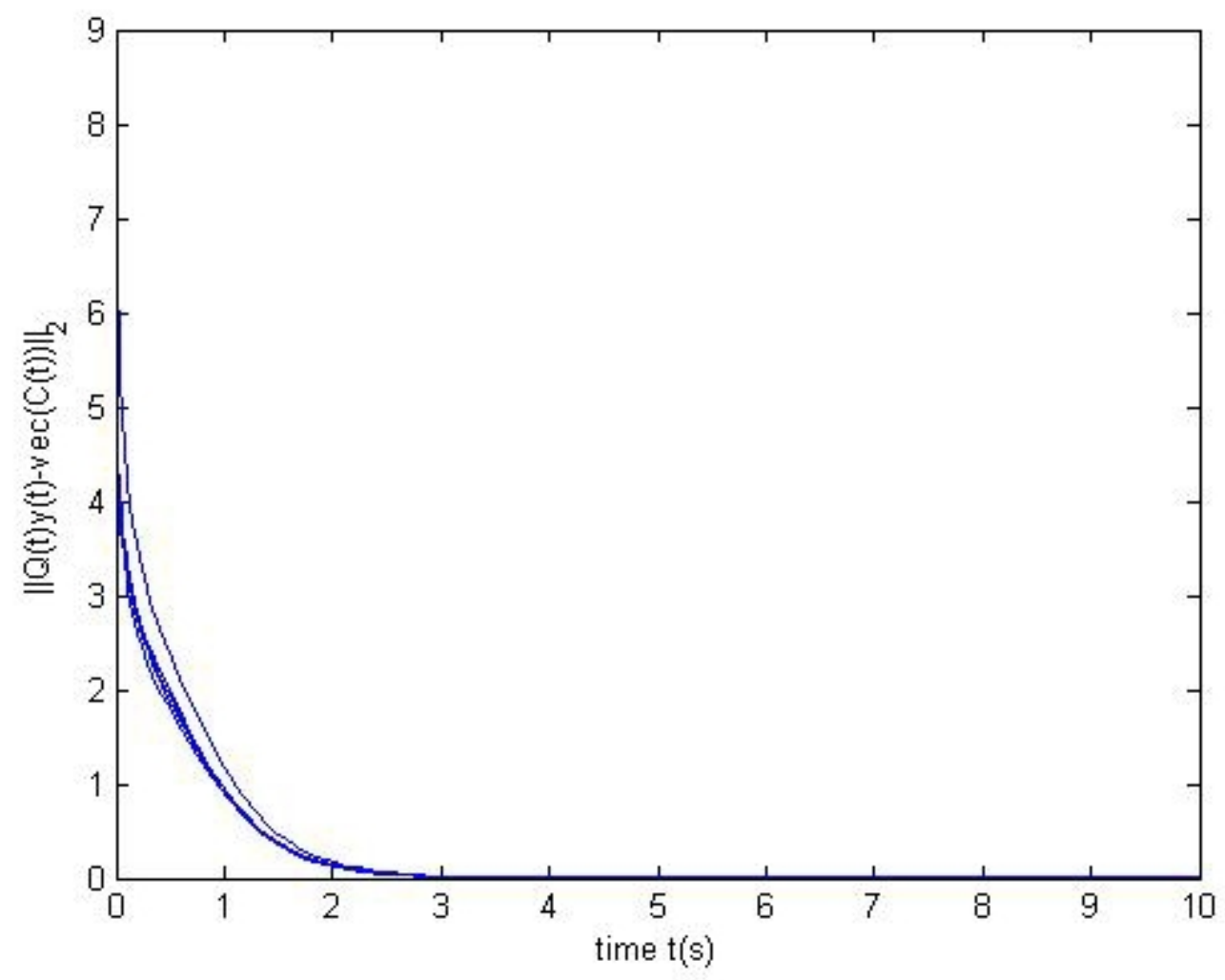

Fig. 2. Residual error $\|Q(t) y(t)-\operatorname{vec}(B(t))\|_{2}$ of the proposed ZNN model for the converted time-varying matrix equation.

These simulation results demonstrate the efficiency of the proposed ZNN model for online Lyapunov matrix inequality.

\section{Conclusions}

In this paper, by introducing a time-varying matrix with non-negative elements, the time-varying Lyapunov matrix inequality (1.2) is converted to a time-varying matrix equation (2.2). With the matrix-valued indefinite error function, the ZNN model (3.3) is established for the time-varying Lyapunov matrix inequality (1.2). Computer simulation results further demonstrate the efficiency and superiority of the proposed ZNN model (3.3) for solving Lyapunov matrix inequality (1.1).

\section{Acknowledgements}

This work was financially supported by National Natural Science Foundation of China (11301330) and the grants of "The First-class Discipline of Universities in Shanghai" and "Gaoyuan Discipline of Shanghai". The authors would also like to thank Prof. Yunong Zhang and his PhD student Dongsheng Guo, School of Information Science and Technology, Sun Yat-sen University, Guangzhou 510006, P.R. China, for their code.

\section{References}

[1] D. Guo and Y. Zhang: Appl. Math. Comput. Vol. 259 (2015), p. 327

[2] E. Semsar-Kazerooni and K. Khorasani: Automatica J. IFAC Vol. 45 (2009), p. 2205

[3] C. Yi, Y. Zhang and D. Guo: Math. Comput. Simulation Vol.92 (2013), p. 40 